\title{
A test of the generality of the word-context effect
}

\author{
JOEL D. SCHENDEL and PETER SHAW \\ University of Illinois at Urbana-Champaign, Champaign, Illinois 61820
}

\begin{abstract}
Three experiments tested the generality of the finding of Reicher (1969) and Wheeler (1970) that a word context facilitated the accuracy of recognition of an embedded target letter. In the present experiments, the context was a letter and the target was a letter fragment. The letter context greatly facilitated the recognition of the letter fragment. In Experiment I, this improvement was in many cases from chance level to perfect performance. An interpretation of this effect in terms of greater familiarity of the fragment and its context was disconfirmed in Experiment II. A procedure of preexposure cueing in Experiment III eliminated the superior recognizability of fragments having a letter context.
\end{abstract}

Reicher (1969) and Wheeler (1970) have reported a result which. apparently, is sufficiently counterintuitive and counter current assumptions to have generated considerable interest. Recognition was found to be better for a target letter embedded in a four-letter word than for the same target letter presented alone for the same brief exposure duration. For most experiments and real-life situations, the redundancy of letter combinations in a word is a possible basis for subjects to guess the target letter. But. under the conditions of the Reicher-Wheeler experiments. a guessing interpretation of their effect appears untenable and the superiority of recognition for a target letter in a word appears to reflect a property of the processing of the letter.

In a typical trial in one of their experiments. the word READ. for example. was presented briefly and then followed by the response alternatives $E$ and $O$. The subject's task was to indicate which letter had appeared in the displayed word. Since ROAD is also a common English word. no clue as to the identity of the target letter is given by knowing any of the letters common to the words READ and ROAD.

Considering the relative recency of Reicher's (Note 1) original report, a considerable amount of research has been produced concerned with identifying the processes underlying the word-context effect (WCE). Little attention, however, has been paid to the question of whether or not the variables responsible for the WCE extend to situations beyond that of a target letter presented in a word or a pseudoword context. Biederman and his associates (Biederman. 1972: Biederman. Glass. \& Stacy. 1973; Biederman, Rabinowitz, Glass. \& Stacy, 1974) have conducted a series of studies which may be relevant to the question of the generality of the WCE. In the

The research was supported by Grant 41-32-66-376 from the Research Foundation of the University of Illinois to the second author. Requests for reprints should be sent to Joel D. Schendel. Department of Psychology. University of Illinois. Champaign. Illinois 61820 .
Biederman (1972) study. the effect of context upon perceptual recognition was tested using photographs of real-world scenes as stimuli. The subject's task was to identify which of four objects that had been presented somewhere in the display was the one which had been presented in the cued position of either a normal photograph (coherent) or a random permutation of rectangular sections of the same photograph (jumbled). The position of the cued object was the same in both the coherent and the jumbled versions of the photograph. Even when the subject knew in advance of stimulus presentation "where to look and what to look for" in the photograph, the accuracy of selecting the cued object in the coherent condition was superior to the accuracy of the jumbled condition.

Basic procedural differences make it difficult to draw direct comparisons between Biederman's findings and those of Reicher (1969) and Wheeler (1970). ${ }^{1}$ Perhaps more relevant to the issue of the generality of the WCE is a study by Weisstein and Harris (1974). Weisstein and Harris. using a four-alternative Reicher-Wheeler forced-choice procedure. observed that a line segment is recognized better when it is presented in the context of a figure that looks unitary and three-dimensional than when the segment is presented in the context of a less coherent "tlat" pattern.

If the variables underlying the WCE are to be of general usefulness for models of the human recognition system, an exacting test of the generality of the WCE is required. The present experiments were conducted. therefore, in an attempt to determine if the effect reported by Reicher and Wheeler for a target letter embedded in a word would also occur at the level of a letter tragment embedded in its letter context. The recognition measure of main interest in Experiment I was response accuracy for letter fragments when they were viewed alone or viewed in their conventional letter contexts. Experiment II tested a familiarity interpretation of the effect obtained in Experiment I. The third experiment 


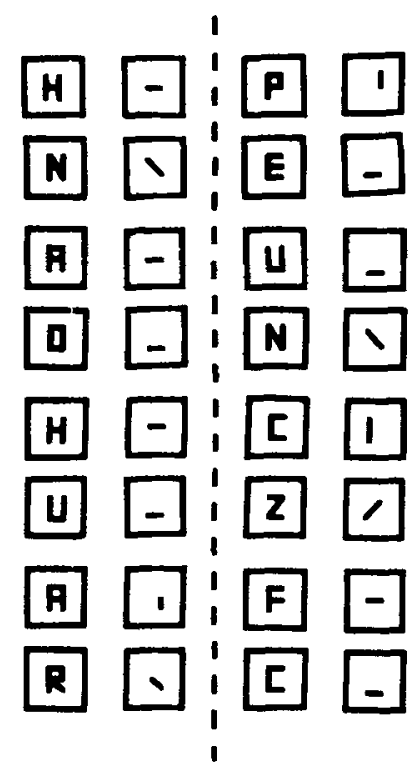

Figure 1. Letter and critical fragment stmulus sets.

differed from the two previous experiments in that the subject was allowed to examine the relevant response alternatives in advance of a series of trials. The studies (e.g.. Bjork \& Estes, 1973; Massaro, 1973; Thompson \& Massaro. 1973) that have combined the essentials of the Reicher-Wheeler procedure with a procedure of preexposure cueing have found recognition performance for a letter embedded in a word to be no better than recognition performance for a single letter display. Experiment III was designed to test this result at the level of a letter fragment embedded in its letter context.

The general procedure used throughout the present research was similar to that employed in the experiments of Reicher (1969) and Wheeler (1970). The task required a forced choice between two alternative letter fragments, one of which appeared in the stimulus. If the stimulus was a letter, the other response alternative in the forced-choice task formed a new letter given the contextual elements of the stimulus. For example, two parallel vertical letter fragments are structurally common to the letter pair " $\mathrm{H}$ " and "N." Each letter is discriminated from its alternative by the position of a single letter fragment (hereafter called the critical fragment). If the letter " $\mathrm{H}$ " was the stimulus, the two alternatives in the forced-choice task were a critical fragment in the horizontal " $\mathrm{H}$ " position and a critical fragment in the alternate-but-equally-likely slanted " $N$ " position. Each fragment stimulus was matched in both size and position to the critical fragment of a corresponding letter stimulus. The stimulus was always either a critical fragment presented alone or presented in its letter context. The response alternatives were always isolated critical fragments.

The eight letter-stimulus pairs are presented in the left half of each panel and the corresponding fragment stimuli are presented in the right half of each panel in Figure 1 (e.g., H-N, A-O, etc.). Each letter and critical fragment display consisted of a letter or fragment outlined by a $22.2 \times 22.2 \mathrm{~mm}$ square. The uppercase rectangular letters were $9.5 \mathrm{~mm}$ high and $6.4 \mathrm{~mm}$ wide and were centered within the square. The response alternative displays were made up as separate displays but resembled a pair of horizontally aligned fragment stimulus displays. Displays were constructed with $1 / 16$-in. $(1.59-\mathrm{mm})$ white matte opaque tape (Zip-A-Line, No. (-61-M) on high-gloss black paper, and were photographed with $35-\mathrm{mm}$ black-and-white highcontrast copy film. The negatives were mounted as slides so that the stimuli appeared as black figures on a bright background. Each stimulus display subtended approximately $2 \mathrm{deg}$ of visual angle. The black focusing bars had a length equal to half the width of a letter and were seen, except when the display was presented, centered immediately above and below the display. A Scientific Prototype Model GB three-channel autotachistoscope was used for stimulus presentation. The stimulus field and the adaptation field had a luminance of $4.3 \mathrm{~L}$, as measured by a Sepctra Spot Photometer. Reaction times were measured via a General Radio Model 1192-B counter in conjunction with a megacycle pulse generator.

\section{EXPERIMENT I}

\section{Method}

Subjects. Two undergraduate and two graduate students at the University of Illinois served as paid volunteers. All subjects were right-handed and had normal vision.

Procedure. Each session began with $3 \mathrm{~min}$ of dark adaptation. The sequence of events for a typical trial is depicted in Figure 2. The subject was instructed to fixate between the focusing bars and, when the bars were properly focused, to trigger stimulus onset with a foot pedal. The stimulus display was followed immediately with the response alternative display which appeared to the right of the original stimulus display and remained on for $1 \mathrm{sec}$. While the response alternatives were being displayed, the subject moved a hand switch either

-

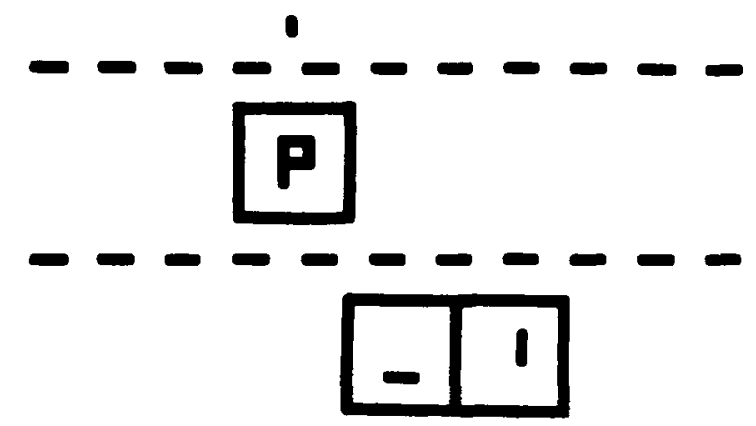

Figure 2. The sequence of events for a typical trial in Experiment I. 
Table 1

Correct Recognition Proportions for each Subject for each of the Eight Letter-Fragment Sets

\begin{tabular}{|c|c|c|c|c|c|c|c|c|c|c|c|c|c|c|c|c|c|c|}
\hline \multirow[b]{3}{*}{ Subject } & \multicolumn{18}{|c|}{ Stimuli: Experiment I } \\
\hline & \multicolumn{2}{|c|}{ H-N } & \multicolumn{2}{|c|}{ U-N } & \multicolumn{2}{|c|}{ O-A } & \multicolumn{2}{|c|}{$A-R$} & \multicolumn{2}{|c|}{$C-Z$} & \multicolumn{2}{|c|}{ C-F } & \multicolumn{2}{|c|}{ U-H } & \multicolumn{2}{|c|}{ P-E } & \multicolumn{2}{|c|}{ Overall } \\
\hline & $\mathrm{F}$ & L & $\mathbf{F}$ & L & $\mathbf{F}$ & $\mathbf{L}$ & $\mathbf{F}$ & L & $\mathbf{F}$ & $\mathbf{L}$ & $\mathbf{F}$ & $\mathbf{L}$ & $F$ & L & $\mathbf{F}$ & $\mathbf{L}$ & & $\mathrm{L}^{*}$ \\
\hline $\begin{array}{l}\text { SS }_{1} \\
\text {. }\end{array}$ & .61 & .98 & .59 & 1.00 & .47 & .99 & .57 & .52 & .60 & .99 & .58 & .99 & .53 & .99 & .58 & .98 & .57 & .93 \\
\hline $\mathrm{JE}_{2}$ & .69 & .81 & .68 & .98 & .58 & .99 & .47 & .52 & .64 & .94 & .59 & .98 & .55 & .94 & .54 & .95 & .59 & .89 \\
\hline $\begin{array}{c}\mathrm{FB} \\
3\end{array}$ & .73 & .86 & .65 & .93 & .66 & .97 & .68 & .54 & .68 & 1.00 & .66 & .99 & .70 & .97 & .81 & .90 & .70 & .90 \\
\hline $\begin{array}{r}\mathrm{NN} \\
4\end{array}$ & .67 & .97 & .58 & 1.00 & .44 & .99 & .67 & .46 & .68 & .97 & .51 & .99 & .58 & .99 & .43 & .99 & .57 & .92 \\
\hline Overall & .68 & .91 & .62 & .98 & .54 & .90 & .60 & .51 & .65 & .98 & .59 & .59 & .59 & .99 & .59 & .97 & .61 & .91 \\
\hline
\end{tabular}

left or right in the direction of the response alternative which he thought had appeared in the stimulus display. The experimenter then recorded the response as well as the response latency and gave the signal, an audible click, for the next trial. Otherwise, the subject paced himself through the task. Each session lasted about $30 \mathrm{~min}$. Initial instructions to the subject emphasized: (a) response accuracy over response latency; (b) that one of the two response alternatives was correct on each trial; and (c) that a response should be given on every trial even if guessing was necessary.

All of the subjects received the same stimulus presentation schedule. An experimental session consisted of six blocks of trials with 32 trials per block. For each session, the letter and fragment stimuli in each block were randomly permuted and the blocks were randomly permuted. The letter and fragment stimuli were randomly ordered across the six blocks with the constraint that a minimum of two stimuli separate successive replications of letter and fragment stimulus presentations. The stimuli were so ordered to minimize the possibility of the subject's anticipating the identity of an upcoming stimulus. Each letter and fragment was presented six times in a session. Across the six presentations of a letter or a fragment stimulus, the correct critical fragment response alternative appeared three times as the left response alternative and three times as the right response alternative. A rest period of approximately $30 \mathrm{sec}$ followed each block, during which time the experimenter changed slide trays.

Each subject participated in three or four practice sessions prior to the eight sessions of data collection. As part of the initial instructions, the experimenter selected five letter and five critical fragment stimuli at random and presented them to the subject with the stimulus exposure duration increased to $5 \mathrm{sec}$. Once the subject understood the task requirements, the stimulus exposure duration was successively lowered from $150 \mathrm{msec}$ to a duration which yielded $75 \%$ accuracy. Knowledge of results was given throughout practice but was terminated once testing began. Exposure durations were lowered whenever necessary during testing to maintain $75 \%$ accuracy. The exposure durations for individual subjects, specified in milliseconds, for Sessions 1 and 8, respectively, were: S.S.1, 25-25; J.E.2, 20-18; F.B. $3,25-8$; and N.N.4, 33-30.

\section{Results and Discussion}

Response accuracy. Table 1 presents the proportions of correct responses for each subject for each of the eight letter-fragment sets. The number of observations for each proportion is 96 , except for a few instances where there were one or two mistrials. A mistrial was counted when the subject indicated that he had inadvertently pushed the hand switch in the $u$ rong direction. It can be seen from Table 1 that recognition accuracy for critical fragments viewed in a letter context is. in general, greatly superior to critical fragments viewed alone. In many cases, the letter context improves accuracy from chance to essentially perfect.

The A-R letter-fragment comparison is the only stimulus set for which the letter-context effect (LCE) is not in evidence. The absence of the LCE may be due to the particular shape of the " $A$ " stimulus context. which several subjects reported appeared more "R-like" than "A-like," Alternatively, the relatively small A-R critical fragments may have been partially or totally masked by the adjacent portions of the letter context. In either event, the fact that the subjects' werall recognition accuracy scores in the A-R letter condition were consistently below their scores under other letter and fragment stimulus conditions clearly indicates that the discriminability of a critical fragment can be reduced as well as enhanced by its letter context.

Response latency. In recognition experiments, where response accuracy is maintained at a level appreciably below perfect performance, a reliable inference from the latency data is quite difficult to make because of the subject's unknown guessing processes. For this reason, the latency data from Experiment I should be treated with caution. The mean latencies for correct responses for the eight letter-fragment sets are presented for each subject in Table 2 . In virtually every case, the LCE is reflected in shorter recognition latencies for critical fragments viewed in their letter contexts.

A further experiment was attempted under $100 \%$ accuracy conditions in order that a more definitive statement with respect to response latencies and the LCE could be made. The procedure was essentially the same as that employed in Experiment I, with two major exceptions. A bonus system was devised to 
Table 2

Mean Latency for Correct Responses in Milliseconds for each Subject for each of the Eight Letter-Fragment Sets; Total Number of Correct Responses are Shown in Parenthesis.

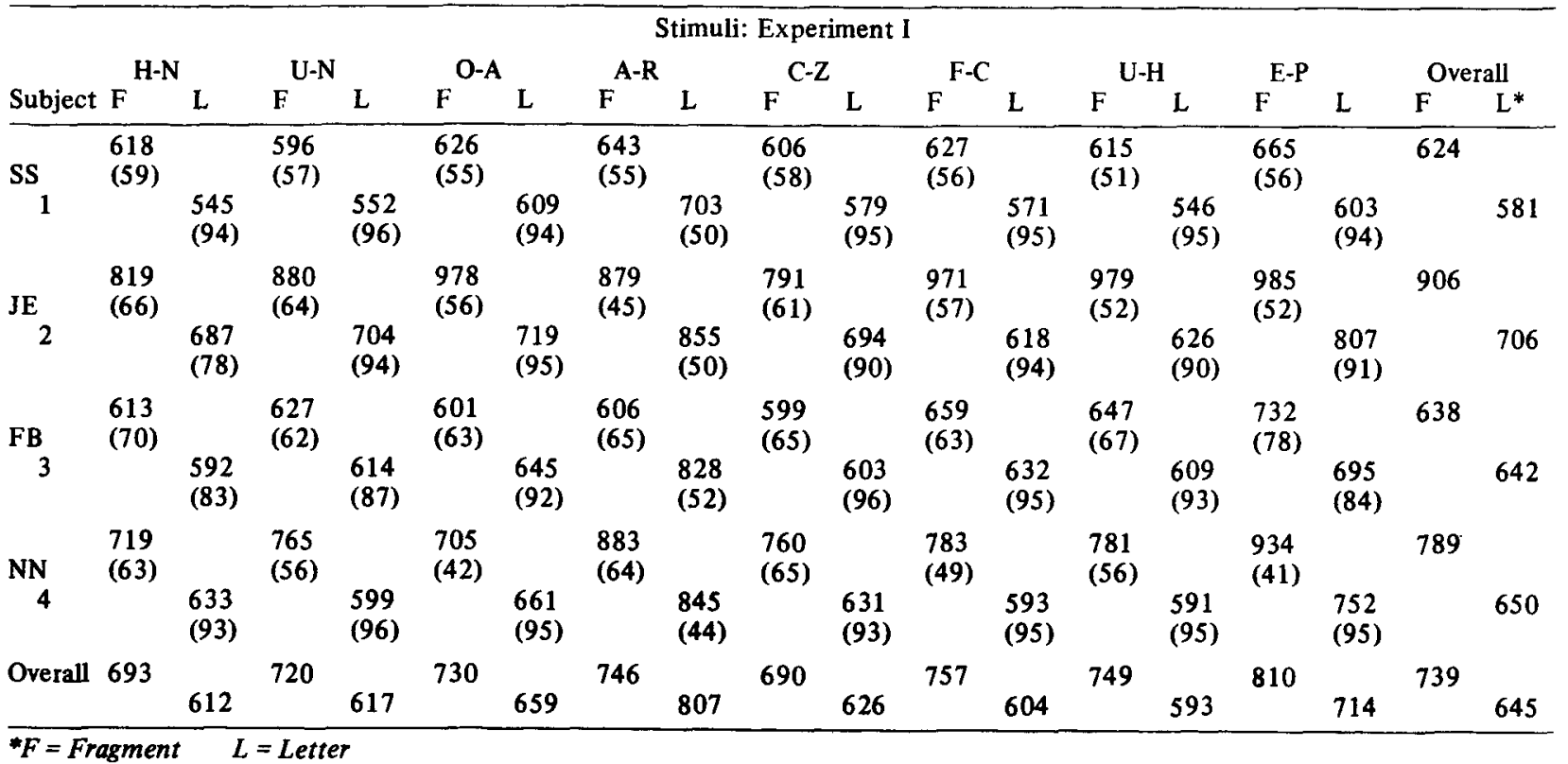

emphasize the importance of short response latencies as well as to discourage errors, and two rather long stimulus exposure durations, 100 and $150 \mathrm{msec}$, were used throughout testing. Unfortunately, no clear statement with regard to recognition latencies could be made from the data. At the 100 -msec exposure duration. shorter response latencies were observed when fragments were embedded in a letter context but recognition errors were still unacceptably high. Very few recognition errors were made at the 150 -msec exposure duration; but the extended exposure duration eliminated any observable effect that the letter context may have had upon recognition latencies.

\section{EXPERIMENT II}

A letter is not commonly thought of as providing a context for letter fragments, but this apparently is the case. One reason a letter is not usually thought of in these terms may be that a line which is to be regarded as letter fragment rarely if ever appears apart from its respective letter context in real-life situations. In the present experiments, subjects were presented a critical fragment either alone or in its usual letter context. Undoubtedly, each subject had experienced line segments similar to the fragment stimuli prior to these experiments. However, it is doubtful that prior to these experiments the subjects had ever been set to expect an isolated "letter fragment" rather than an isolated "line segment." If it can be safely assumed that the subjects' processing reflected the set to regard the critical fragment as an isolated letter fragment. an interesting interpretation of the LCE is possible, Perhaps the facilitating effect of embedding a critical fragment in a letter is the result of the subjects' greater preexperinental experience with the letter fragment in its letter context than with the same fragment in a blank context. In other words, the "familiarity" of the critical fragment and its context as a visual unit may be an important variable for the LCE.

The WCE finding of Reicher (1969) and Wheeler (1970) that a letter is perceived more accurately when it appears in a word than when it appears alone and the finding of Reicher (1969) that a letter embedded in a word is recognized better than a letter embedded in a nonsense quadrigram are consistent with an interpretation of the LCE in terms of a difference in familiarity for conditions. In the case of the WCE, this interpretation is based on the understanding that the familiarity of a letter in any background is not relevant. What is relevant is the familiarity of the visual unit-a letter in a blank background vs. a letter in a word context or a letter in a nonword context vs. a letter in a word context. Clearly, subjects experience letters more often in a word context than in an empty field or nonword context.

If the facilitating effect of embedding the critical fragment in its letter context is attributable to differences in the familiarity of the critical fragment and context visual unit, then it should be possible to reduce substantially if not eliminate the LCE by reducing the difference in the familiarity of the critical fragment and context between the two experimental conditions. An attempt to manipulate the variable of 
familiarity was made in Experiment II. The subjects were required to recognize letter and fragment stimuli which had been rotated $90 \mathrm{deg}$ in either a clockwise or a counterclockwise direction. Since alphabetic characters are almost always experienced in one conventional upright orientation, rotating the character would, under some assumptions, make the critical fragment and its letter context sufficiently unfamiliar to the subject to eliminate most of the LCE found in Experiment 1 .

\section{Method}

Subjects. The same subjects participated as in Experiment I, with the exception of N.N.

Procedure. The stimulus sets selected for use in Experiment II were: P-E, C-F, C-Z, and U-N. The main consideration in stimulus selection was to avoid letters that were essentially unchanged by clockwise and counterclockwise rotations (e.g., " $O$ "). The rotated letter stimulus displays and an example of a rotated response alternative display for the P-E decision are presented in Figure 3.

Depending upon the condition of rotation, the response alternatives appeared immediately above (counterclockwise) or below (clockwise) the stimulus display. Since the two response alternatives were now aligned vertically with the stimulus, the hand switch was adjusted so that it could be moved either up or down in the direction of the correct response alternative. The same focusing bars were used as in Experiment $I$, but they too were rotated $90 \mathrm{deg}$ clockwise or counterclockwise depending upon the test condition. The stimuli used during Experiment II were arranged according to the same criteria as were used in Experiment I.

Four practice sessions preceded 12 sessions of data collection. The same procedure was used throughout practice and testing as was used in Experiment I with but three exceptions: (a) the stimulus exposure duration was successively lowered from 200 msec during the practice sessions; (b) the stimulus displays were rotated in a clockwise direction on odd-numbered sessions and in a counterclockwise direction on even-numbered sessions throughout both the practice and data collection sessions; and (c) each session was divided into six blocks of trials with 16 trials per block.

Individual stimulus exposure durations, specified in milliseconds, for Session 1 and Session 12, respectively, were: S.S.1, 40-20; J.E., 30-22; and F.B. 3, 7-6.5.

\section{Results and Discussion}

The correct recognition proportions for each subject for each of the four letter-fragment sẹts for

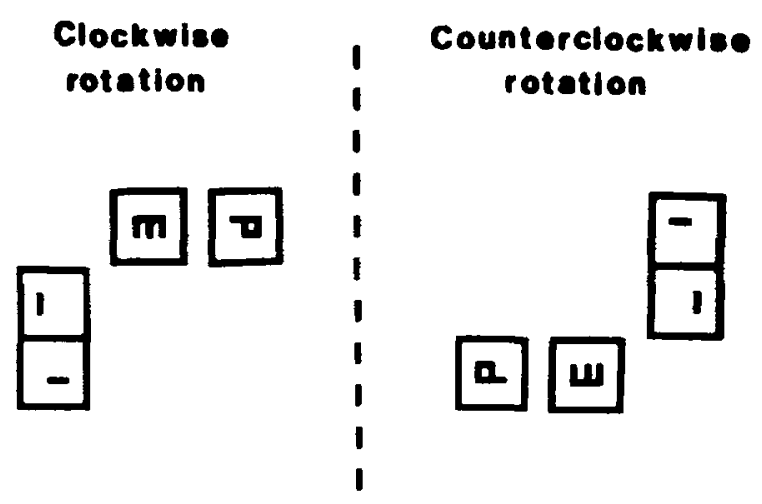

Figure 3. Clockwise and counterclockwise atimulus rotations for the P-E decision.

both clockwise and counterclockwise rotations are presented in Table 3. The number of observations for each proportion is 96 except for a few instances where there were one or two mistrials.

Rotating the critical fragment and its context had little overall effect upon the subjects' recognition performance. Recognition performance was found to be appreciably better when the critical fragment appeared in a rotated letter context than when it appeared alone. In many cases, the - effect of embedding the critical fragment in a rotated letter context was. as in Experiment $I$, to improve recognition accuracy scores from nearly chance to nearly perfect.

The recognition accuracy scores suggest either that the LCE is not attributable to differences in the familiarity of a critical fragment and its context as a visual unit or that rotation does not appreciably reduce the familiarity of alphabetic characters. At this point there seems to be more convincing evidence in favor of the former interpretation. Perhaps the strongest evidence against the argument that rotation did not affect the recognition of letter stimuli is the fact that the average exposure duration giving $75 \%$ accuracy was longer for two of the three subjects in Experiment II than for the same subjects in Experiment 1. This was true even though these

Table 3

Correct Recognition Proportions for each Subject According to Stimulus Set and Rotation Condition

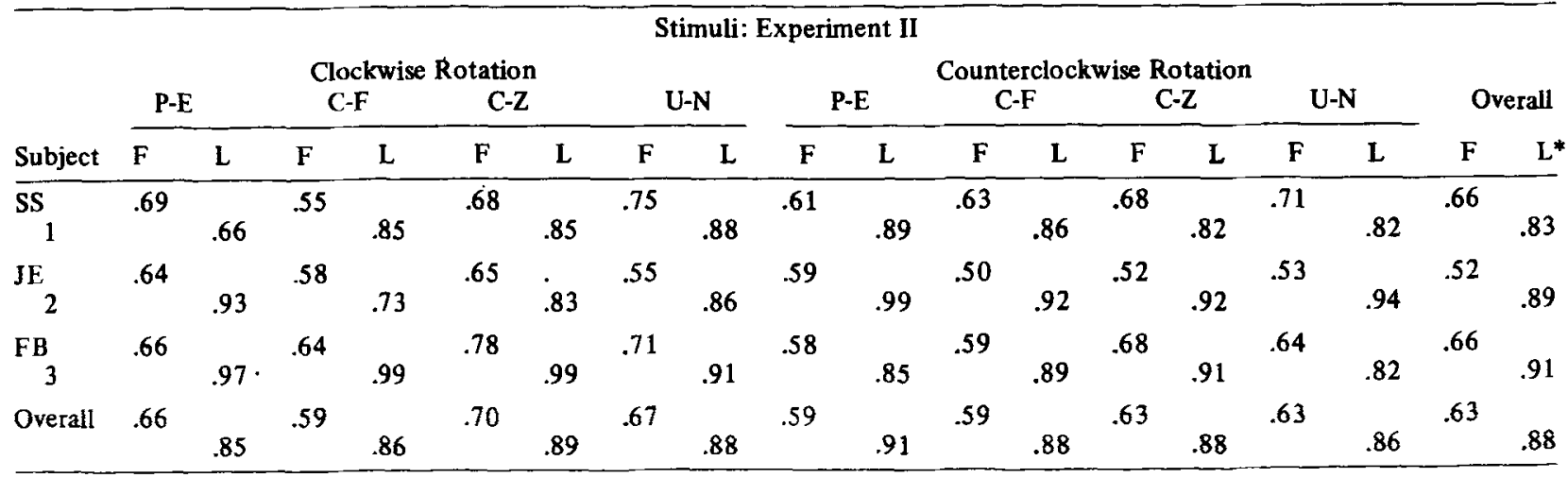

*F= Fragment $L=$ Letter 
subjects pariticpated in no fewer than 19 experimental sessions prior to Experiment II and an additional four practice sessions under the Experiment II conditions prior to testing.

\section{EXPERIMENT III}

Bjork and Estes (1973) and others (e.g., Massaro, 1973; Thompson \& Massaro, 1973) have contributed to an understanding of what, at first glance, appear to be conflicting findings in the research concerned with the visual processing of character displays. On the one hand, there is the well-documented finding that when a subject is asked to scan an array of characters in search of a target character or set of characters, recognition accuracy for the target character decreases as the number of additional characters concurrently displayed increases (Estes, 1972). On the other hand, studies such as those by Reicher (1969) and Wheeler (1970), where the subject is informed as to the identity of the relevant alternatives for a particular trial by a postexposure cue, consistently show that recognition accuracy for a target letter is increased if it is viewed in the context of a word rather than viewed alone.

A major concern of the Bjork and Estes (1973) study was to determine whether the apparently conflicting results arising from these two lines of research were attributable to the pre- vs. postexposure cueing difference in the two procedures. To test this hypothesis, Bjork and Estes (Experiment I) utilized the essentials of the Reicher-Wheeler two-alternative forced-choice procedure but informed the subjects in advance of a series of trials as to the identity of the relevant response alternatives. Under a procedure of preexposure cueing, the identifiability of letters embedded in words was never better than isolated letters and was, in fact, significantly worse than the identifiability of isolated letters when the stimulus exposure durations were reduced and pre- and postexposure masks were introduced. The purpose of Experiment III was to test the findings of Bjork and Estes for preexposure cueing at the level of a critical fragment embedded in its letter context.

\section{Method}

Subjects. Four new undergraduates from the University of Illinois served as paid volunteers. K.E. was able only to complete the first phase of testing. All subjects were right-handed and had normal vision.

Procedure. The stimulus materials were the same as in Experiment 1, with the exception that the A-R letter-fragment set was omitted. Experiment III was carried out in five separate phases.

Postexposure cueing. Phase I of the experiment was conducted in the same manner as in Experiment $I$ with but two exceptions. Each subject participated in two rather than eight data collection sessions, and each session consisted of six blocks of trials with 28 trials per block. Exposure durations giving $75 \%$ accuracy for each subject for the two sessions under Phase I, specified in terms of milliseconds, were as follows: L.C..1, 22-20; T.R.2, 10-8; S.H.3, 10-12; K.E.4, 22-20.
Preexposure cueing. Several general procedural changes distinguished the four latter phases of testing from the Phase I procedure, the major procedural change being the provision for preexposure cueing. Each of the seven letter-fragment sets was presented in two blocks with 12 trials per block. The stimuli that were members of a letter-fragment set were randomly ordered across the two blocks. Within a block, the response alternatives were always the same two critical fragments and each critical fragment was always in the same left-right position. Across a two-block set, the left-right positions of the two critical fragment response alternatives were interchanged.

At the beginning of each block, the subject was presented the response alternative display type for that block for a period of $10 \mathrm{sec}$ and was told to memorize the response alternatives as they were presented in the display. The sequence of events for a typical trial was programmed such that the following events occurred when the subject initiated the trial: (a) as a trial-by-trial reminder, each trial began with a 4-sec presentation of the response alternative display for that block; (b) the response alternatives were immediately followed by the focusing bars which were presented for $750 \mathrm{msec}$; (c) the stimulus was then presented and the subject gave his response.

All of the subjects received the same stimulus presentation schedule. The seven letter-fragment sets were presented in a different random order each session. The order of the blocks in each two-block set was alternated and the stimuli in each block were randomly permuted after each session. Response alternative displays were also switched between blocks after each session.

Phases II through $V$ differed with respect to the stimulus exposure durations used: (a) Phase II was a post- to preexposure cueing transfer test. A single session was conducted during which the stimuli were presented at the $75 \%$ accuracy exposure duration that each subject had established during the last session of Phase I. (b) Prior to Phase III, each subject participated in a single session during which the stimulus exposure duration was successively lowered from the Phase I and Phase II $75 \%$ accuracy duration to a duration which yielded $75 \%$ accuracy under the preexposure cueing technique. Phase III had four sessions during which the stimulus exposure duration was adjusted after each session in order to yield a new duration which gave $\mathbf{7 5 \%}$ accuracy for each subject as his performance improved. Starting and finishing exposure durations in milliseconds for each subject during Phase III were: L.C.1, 4.5-3.5; T.R.2, 3-2.85; S.H. 3, 2.7-2.4. (c) Phase IV consisted of a single session during which the performance of each subject was measured after $2 \mathrm{msec}$ had been added to the $75 \%$ accuracy exposure duration established during the final session of Phase III. (d) Phase $V$ consisted of a single session during which performance was measured after $1 \mathrm{msec}$ had been subtracted from the $75 \%$ accuracy exposure duration established during the final session of Phase III.

\section{Results and Discussion}

Correct recognition proportions are summarized for letter and fragment stimuli for each subject by test phase in Table 4. Except for a few instances where there were one or two mistrials, the number of observations per proportion in Phase I through Phase $V$ are as follows: $168,84,336,84$, and 84 .

Phase I. Under the postexposure cueing procedure, the subjects showed the same strong LCE that was demonstrated in Experiment I.

Phase II. When the Phase I 75\% accuracy exposure durations were used but the subject was given advance information as to the identity of the alternatives to be tested over a block of trials, overall 
Table 4

Correct Reocgnition Proportions for each Subject for Letter and Fragment Stimuli by Experiment II Test Phase

\begin{tabular}{|c|c|c|c|c|c|c|c|c|c|c|}
\hline \multirow[b]{2}{*}{ Subject } & \multicolumn{2}{|c|}{ Phase I } & \multicolumn{2}{|c|}{ Phase II } & \multicolumn{2}{|c|}{ Phase III } & \multicolumn{2}{|c|}{ Phase IV } & \multicolumn{2}{|c|}{ Phave V } \\
\hline & I: & $\mathbf{L}$ & $1:$ & $\mathbf{L}$ & I: & $\mathbf{L}$ & $F$ & $\mathbf{L}$ & $\mathbf{F}$ & $\mathbf{L}^{*}$ \\
\hline $\begin{array}{l}\text { LC } \\
1\end{array}$ & .67 & .98 & .99 & 1.00 & .81 & .69 & .98 & .96 & .63 & .50 \\
\hline $\begin{array}{r}\mathrm{TR} \\
2\end{array}$ & .71 & .95 & 1.00 & 1.00 & .74 & .63 & 1.00 & .98 & .57 & .60 \\
\hline $\begin{array}{r}\text { SH } \\
\quad\end{array}$ & .61 & .88 & 1.00 & .99 & .79 & .70 & 1.00 & .93 & .50 & .46 \\
\hline $\begin{array}{r}\mathrm{KI} \\
4\end{array}$ & .59 & .97 & & & & & & & & \\
\hline Overall & .65 & .95 & .99 & .99 & .78 & .67 & .99 & .96 & .57 & .52 \\
\hline
\end{tabular}

recognition performance was virtually perfect for each subject. Such a result has something to say for the perceptual processing of character displays in general. One possible explanation for the Phase II results is that preexposure cueing enables the subject to set up a feature extraction program which would assure that ficatures most relevant to the identification of the input pattern for the current block are extracted. The improved performance after preexposure cueing might then be attributed to the fact that the features most relevant to the identification of the stimulus are nore apt to be extracted with preexposure cueing that without preexposure cueing.

Phase III. A separate two-tailed $Z$ test comparing the proportions correct for isolated critical fragments and critical fragments embedded in letters was computed for each subject. A substantial advantage in the identifiability of critical fragments presented alone over critical fragments embedded in letters was indicated. For each of the subjects, the $\mathrm{Z}$ test was found to be significant $\left(\mathrm{Z}_{1}=3.66 ; \mathrm{Z}_{2}=3.01 ; \mathrm{Z}_{3}=\right.$ $2.69)$ beyond an alpha level of .01 . This finding is consistent with Bjork and Estes' (1973) earlier finding that isolated letters are recognized better than letters embedded in words under preexposure cueing and visually degraded conditions.

Phase IV and Phase V. The latter two phases of Experiment III were exploratory in nature. Phase IV tested the effect of preexposure cueing under slightly less demanding conditions than Phase III. No advantage in recognition performance was found when the critical fragment was embedded in its letter context for L.C. ${ }_{1}$ and T.R. ${ }_{2}$. S.H. ${ }_{3}$, however, showed superior recognition performance for isolated fragments, $Z_{3}=2.48, p<.05$, during Phase IV. Phase $V$ tested the effect of preexposure cueing under conditions which were considered to be extremely demanding. Not surprisingly, recognition accuracy scores were at chance level for both stimulus types.

Summary. To brietly summarize the important findings of Experiment III: (a) the LCE appeared only under a postexposure cueing procedure; (b) preexposure cueing resulted in near perfect recognition accuracy for both letter and fragment stimuli; and (c) under conditions of preexposure cueing and reduced exposure durations, recognition accuracy for a critical fragment presented alone was appreciably better than recognition accuracy for a critical fragment embedded in a letter context.

\section{GENERAL DISCUSSION}

Under a procedure similar to that used by Reicher $(1969)$ and Whecler (1970), recognition performance was found to be significantly better for displays having a critical fragment embedded in a letter context than for displays having the eritical fragment presented alone. This finding suggests that the effect reported by Reicher and Wheeler holds for a larger class of situations than just the perception of a target letter embedded in a word. It adds a further test of the adequacy of any theoretical account of the WCE: Does the theory also account for the LCE as well?

The WCE has been attributed to differences between conditions with respect to four theoretical variables: (1) frequency of a stimulus in past experience as an undivided visual unit (familiarity); (2) ease and/or probability of producing an auditory name for the stimulus (pronounceability); (3) number of leature detectors evoked by the stimulus (more leatures); (4) amount of advantage given the discriminating features of the stimulus in terms of either processing priority or processing weight.

\section{Familiarity}

A familiarity interpretation of the LCE was tested in Experiment 11. An appreciable advantage was lound in the identifiability of critical fragments embedded in rotated letter forms over critical fragments presented alone. This result indicates that either the familiarity of the critical fragment and its context is not an important variable for the LCE or 
that rotation did not substantially reduce the faniliarity of the letters. The generally longer $75 \%$ accuracy exposure durations under Experiment II lent some support to the former interpretation.

When these findings are considered in relation with the findings from the WCE literature. a familiarity interpretation of the LCE is questionable. Baron and Thurston (1973) and Herrmann and McLaughlin (1973) show'ed that the WCE holds up just as strongly for pronounceable nonwords as for words. This finding rules out a familiarity interpretation of the WCE, assuming that a letter embedded in a nonword is unfamiliar to a subject.

\section{Pronounceability}

Herrmann and McLaughlin (1973) showed that a target letter embedded in a letter string which conforms to the rules and regularities of English spelling is detected better than the same target letter in a corresponding. but randomly arranged, string of letters. On the surface, it appears as if the WCE is critically dependent upon the subject's knowledge of English spelling patterns. However, letter strings which conform to the spelling patterns of English (e.g. " "GLART") are also clearly more pronounceable than randomly arranged strings of letters (e.g., "TGALR"). Therefore, an alternative interpretation of the WCE is possible: the pronounceability of the letter string may account for the WCE.

Perhaps the strongest support for a pronounceability interpretation of the WCE comes from Mezrich (1973). Mezrich's study incorporated the essentials of the Reicher-Wheeler paradigm with the exception that experimental subjects were required to vocalize the stimulus during a delay period between the offset of the stimulus and the onset of the response alternatives. Even if the subject had no idea what the stimulus was, he was "to say something" before the response alternatives were presented. Control subjects performed the task in the usual way without rocalization. Consistent with the predictions of the pronounceability hypothesis. control subjects showed the WCE whereas the "vocalizers" did not.

Perhaps the best evidence that has been cited against a pronounceability hypothesis is Wheeler's (1970) tinding that words were superior to isolated letters even though both would seem to be equally pronounceable and Gibson. Shurcliff, and Yonas' (1970) finding that. in a free-report paradigm, pronounceable stimuli retain their advantage even for the congenitally deaf. In the instance of the Gibson et al. study, one would have to argue that their congenitally deaf subjects were, in fact, pronouncing the stimuli to be consistent with the pronounceability hypothesis (see Baron \& Thurston, 1973).

Viewed by itself. the present research is consistent with the pronounceability hypothesis. There is no question that the letter stimuli are pronounceable. whereas this is clearly not the case for the isolated critical fragments. The present results are puzzling. lowever. if the pronounceability interpretation is correct. To support the pronounceability interpretattion. one would have to argue that pronounceability enhanced the identifiability of the letter stimuli in the current study but not in Wheeler's (1970) study. Such an argument seems, at best. difficult to support and perhaps not worth making.

\section{More Features}

The interpretation of the WCE in terms of "more feature" "is based upon the assumption that pattern recognition is a process of testing the visual input for the presence of particular parts or properties. Numerous models of perceptual recognition and memory are based upon such an assumption. At the lower level of Selfridge's (1966) "Pandemonium," for example, are the "feature demons" which look for the presence of particular characteristics in the pattern: lines. curves. angles, and so on. Several mechanisms in other models such as Hebb's (1949) "cell assembly," the various decision nodes of Feigenbaum's (1963) EPAM, the "operators" of Uhr's (1966) and Uhr and Vossler's (1963) simulation model, and the "components" of Bower's (1967). and Rumelhart's (1970) multicomponent theories are all feature oriented in nature.

Wheeler (1970) has proposed an interpretation of the WCE set in a feature theory framework which seems applicable to the present data. According to feature theories, patterns-in this case, alphabetic characters-are made up of bundles of features. Pattern recognition requires that the features be extracted and subjected to a decision process for identification. If. as in a tachistoscopic recognition situation, processing time is limited, an insufticient number of features may be extracted from each bundle to make an unambiguous identification of the stimulus.

The specific version of the feature extraction process discussed by Wheeler postulates that the extraction of features from all bundles proceeds in parallel. Thus, whenever the word ROAD is briefly exposed or the letter $O$ is presented alone, the proportion of features extracted from the bundle for the target letter $O$ should be the same in each case. Yet, while the amount of information should be the same, recognition performance is substantially better if the target letter is embedded in a word than if it appears alone. What accounts for the performance difference?

Wheeler suggested the possibility that more features relevant to the identification of the target letter might be extracted when the target is embedded in a word than when the target appears alone. Several possible sources of these additional features were discussed by Wheeler: overall shapes of words, shapes 
of letter digrams or trigrams, and spaces between letters. While the overall shapes of words and shapes of letter digrams or trigrams may supply additional features to the subject, and, possibly, may enhance the recognizability of a target letter embedded in a word context, there are some crucial problems with Wheeler's suggestion that the properties of the spaces in a word facilitate the recognition of an embedded target letter. In particular, Reicher's (1969) finding that the letters in a nonsense quadrigram are processed no better than an isolated letter is inconsistent with this interpretation unless one accepts the peculiar assumption that only familiar interletter gaps can provide additional features to the perceptual system. The same point can be made in regard to Johnston and McClelland's (1973) finding that the identifiability of a letter embedded in a series of "\#" signs is no better than the same letter presented alone.

It is not difficult to identify potential sources of additional features in the letter stimuli of the present experiments. The different angles and gaps which distinguish letters may facilitate the recognition of the embedded critical fragment. For illustration, the letter " $\mathrm{H}$ " has a gap at the top and a gap at the bottom. Among the letter choices included in these experiments, the only other letter choice with these characteristics is the letter "N." The letter " $N$ " and the letter " $\mathrm{H}$ " could be easily distinguished from one another, however, if the subject perceived either a right angle or an acute angle in the stimulus. The regular nature of gaps and angles within letters compared to the less regular relations between letters may account for the relatively greater magnitude of the LCE compared to the WCE. While the WCE is often found to be in the $5 \%$ to $8 \%$ range. the LCE was found to be in the $30 \%$ range (see Table 1), and this may even be an underestimate given ceiling effects in the letter conditions.

\section{Processing Priority or Processing Weight}

An interpretation of the WCE in terms of processing priority or processing weight holds that the WCE is the product of the subject's experientially built-in processing biases. Processing may be more effective because the subject gives processing priority to certain feature tests over others or because the subject weights certain feature tests more heavily in a decision process than others. In either case, processing is held to be more efficacious because the subject's past experience predisposes him to process in a way which is advantageous in a certain situation.

At the level of words, the subject's knowledge of the redundant nature of letter-to-letter sequences in English text is assumed to predispose him to test for the presence of certain letters in words earlier than others. In the READ vs. ROAD decision, for example, recognition of the letter $E$ (or $O$ ) is assumed to be facilitated because the subject's past experience with the sequential dependencies of letters in words leads him to test for the presence of letter combinations like READ or ROAD earlier than say a combination like RZAD. Processing is assumed to show greater facilitation as letter-to-letter sequences conform more closely to the subject's biases. Evidence supporting the hypothesis that recognition accuracy increases with transitional probability levels for nonword letter strings comes from Herrmann and McLaughlin (1973). In the case of a target letter presented alone or presented in a nonsense quadrigram, it is unlikely that the subject's processing biases would facilitate recognition performance. In stimulus situations which only randomly conform to the subject's biases, the probability of giving the most relevant feature tests high processing priority or processing weight would be essentially chance.

In terms of the present research, the letter context may have served a function analogous to that of the spelling pattern in the Reicher-Wheeler studies. Given. for instance, two parallel vertical letter fragments, as in the letter " $H$," the subject's experience with letters may have predisposed him to test for the presence of a connecting horizontal earlier than for the presence of a letter fragment which would have changed the figure into a nonletter. In a similar way, recognition performance for a critical fragment in a blank context may have been comparably worse because chance determined whether the most relevant feature tests received high processing priority or processing weight. The findings for preexposure cueing (Experiment III) are consistent with such an interpretation. A preexposure cue may have made it equally likely under the two stimulus conditions that feature tests most relevant to the recognition of a critical fragment were given greater processing priority or processing weight.

\section{Other Interpretations for the Letter-Context Effect}

Relative vs. absolute judgments. One of our reviewers suggested that embedding a critical fragment within other letter fragments permitted the use of "relative features" (i.e., reference or anchor points) to a much greater degree in the letter conditions than in the fragment conditions. According to this argument, the presence of these reference points afforded the subjects greater opportunity to make relative judgments about the height. length, and angle of the critical fragments tmbedded in letters than of the height, etc., of critical fragments presented alone.

An interpretation of the LCE in terms of "relative is. absolute judgments" is perhaps applicable to the A-O, H-U, and F-C data. For these letter-fragment sets, the subject's response may have depended upon a relative/absolute judgment of the height of the 
Table 5

Correct Recognition Proportions for each Subject for Letter and Fragment Stimuli by Focusing Bar Condition

\begin{tabular}{|c|c|c|c|c|}
\hline \multirow[b]{2}{*}{ Subject } & \multicolumn{2}{|c|}{ Focusing Bars } & \multicolumn{2}{|c|}{ No Focusing Bars } \\
\hline & $\mathrm{F}$ & $\mathbf{L}$ & $\mathbf{F}$ & $\mathbf{L}^{*}$ \\
\hline $\begin{array}{l}\text { GS } \\
1\end{array}$ & .58 & .95 & .60 & .92 \\
\hline $\begin{array}{r}\mathrm{SH}_{2} \\
\end{array}$ & .61 & .87 & .68 & .84 \\
\hline $\begin{array}{r}\mathrm{MH} \\
3\end{array}$ & .54 & .98 & .50 & .98 \\
\hline $\mathrm{JO}_{4}$ & & & .62 & .94 \\
\hline Overall & .58 & .93 & .60 & .92 \\
\hline
\end{tabular}

${ }^{*} F=$ Fragment $L=$ Letter

critical fragment. Such an interpretation does not seem viable for the H-N, P-E, U-N, or C-Z letter-fragment sets, however, where the subject was not required to make absolute judgments along a single continuum. For these stimuli. the length and angle as well as the height of the critical fragment generally all changed. rendering any possible advantage of making relative over absolute judgments unimportant.

Focusing bars. A second possibility, offered by a reviewer. is that the LCE may have been due to a differentially high forward masking effect of the focusing bars upon the isolated critical fragments. To test this hypothesis. four student volunteers were tested under conditions identical to Experiment I with the following exceptions. (a) Each session consisted of six blocks of trials with 28 trials per block, the A-R letter-fragment set being omitted. (b) Prior to testing, each subject participated in one or two practice sessions $\left(P_{1}\right)$ to establish his $75 \%$ accuracy exposure duration. (c) Focusing bars were either present (G.S.1, S.H.2) or not present (M.H.3, J.O.) during $P_{1}$. depending upon whether the bars were to be provided during Test $1 \quad\left(T_{1}\right)$. (d) Following $T_{1}$-a single session-G.S. ${ }_{1}$, S.H.2 ${ }_{2}$, and M.H. ${ }_{3}$, participated in one or two practice sessions $\left(\mathrm{P}_{2}\right)$ designed to obtain their $75 \%$ accuracy exposure durations under focusing bar conditions opposite of those employed during $P_{1}$ and $T_{1}$. (e) A single test session- $T_{2}$-was then conducted during which the $P_{2}$ focusing-bar conditions were maintained for the subject. Exposure durations yielding $75 \%$ accuracy for each subject during $T_{1}$ and $T_{2}$, specified in terms of milliseconds, were as follows: G.S.1, 18-25; S.H.2, 15-25; M.H. 29-25; J.O. . 13.

Table 5 presents the correct recognition proportions for each subject for the letter and fragment stimuli when the focusing bars were either present or absent. The number of observations for each proportion is 84 except for a few instances where there were one or two mistrials. Clearly, it is reasonable to conclude from these data that the focusing bars were not responsible for the LCE. In fact, the $75 \%$ accuracy exposure durations obtained for subjects during $T_{1}$ and $T_{2}$ suggest that the task was simpler when the focusing bars were present than when they were absent.

\section{Conclusion}

The main purpose of the present experiments was merely to give an exacting test of the generality of the WCE. This has been done with a positive result-the LCE. This new effect confirms the hypothesis that there is a general contextual phenomenon: recognition of a component in a display can be facilitated by the presence of the background context.

It is likely that an empirical sentence context effect-superior recognition for a target word in a sentence over an isolated target word-can be obtained. The stimuli in an experiment to show such an effect necessarily must be presented over a period of several hundred milliseconds. In this case, lacking a general theory. it is difficult intuitively to decide whether such an empirical effect should be regarded as another instance of the general contextual phenomenon revealed by the WCE and the LCE. Even more difficult is the problen of what conditions for a paragraph context effect would indicate that it is part of the general context phenomenon.

\section{REFERENCE NOTE}

1. Reicher. G. M. Perceptual recognition as a function of meaningfulness of stimulus material. Technical Report No. 7 . The University of Michigan. Human Performance Center. $1 \% 8$.

\section{REFERENCES}

Baron. J.. \& Thurston, I. An analysis of the wordsuperiority effect. Cognitive Psychology, 1973. 4, 207-228.

Biederman. I. Perceiving real-world scenes. Science, 1972, 177. $77-80$.

Biederman, I., Glass, A. L., \& Stacy, E. W., Jr. Searching for objects in real-world scenes. Journal of Experimental Psychology, 1973, 97, 22-27.

Biederman, I., Rabinowitz, J. C., Glass, A. L., \& Stacy, E. W., JR. On the information extracted from a glance at a scene. Journal of Experimental Psychology, 1974, 103, 597-600.

Bjork, E. L.. \& Estes, W. K. Letter identification in relation to linguistic context and masking conditions. Memory \& Cognition, 1973, 1, 217-223.

Bower. G. H. A multicomponent theory of the memory trace. In K. W. Spence \& J. T. Spence (Eds.). The psychology of leaming and motivation (Vol. I). New York: Academic Press. 1967

EsTES, W. K. Interactions of signal and background variables in visual processing. Perception \& Psychophysics. 1972, 12. 278-286.

Feigenbaum, E. A. The simulation of verbal learning behavior. In E. A. Feigenbaum \& J. Feldman (Eds.), Computers and thought. New York: McGraw-Hill, 1963. 
Gibson, E. J., Shurcliff, A., \& Yonas, A. Utilization of spelling patterns by deaf and hearing subjects. In $H$. Levin \& J. P. Williams (Eds.), Basic studies on reading. New York: Basic Books, 1970.

Heвв, D. O. The organization of behavior. New York: Wiley, 1949.

Herrmann, D. J.. \& Mclaughlin, J. P. Language habits and detection in very short-term memory. Perception \& Psychophysics, 1973, 14, 483-486.

Johnston, J. C., \& MCCLelland, J. L. Visual factors in word perception. Perception \& Psychophysics, 1973, 14, 365-370.

MAssaro. D. W. Perception of letters, words, and nonwords. Journal of Experimental Psychology, 1973, 100, 349-353.

Mezrich, J. J. The word superiority effect in brief visual displays: Elimination by vocalization. Perception \& Psychophysics. 1973, 13. 45-48.

Reicher. G. M. Perceptual recognition as a function of meaningfulness of stimulus material. Journal of Experimental Psychology, 1969, 81, 275-280.

RumelhaRT, D. E. A multicomponent theory of the perception of briefly presented displays. Journal of Mathematical Psychology. 1970. 7, 191.218.

Selfridge, O. G. Pandemonium: A paradigm for learning. In L. Uhr (Ed.), Pattern recognition. New York: Wiley, 1966.

ThOMPSON. M. C., \& MASSARo, D. W. Visual information redundancy in reading. Joumal of Experimental Psychology. 1973, 98. 49-54.
UHR, L. Pattern recognition. In L. Uhr (Ed.), Pattern recognition. New York: Wiley, 1966.

Uhr, L., \& Vossıer. C. A pattern-recognition program that generates. evaluates and adjusts its own operators. In E. A. Feigenbaum \& J. Feldman (Eds.), Computers and thought. New York: McGraw-Hill, 1963.

Weisstein, N., \& Harris, C. S. Visual detection of line segments: A.n object-superiority effect. Science, 1974, 186. 752-755.

WhEeler, D. D. Processes in word recognition. Cognitive Psychology, 1970, 1, 59-85.

\section{NOTE}

1. In the Reicher-Wheeler experiments, the subject was required to make a forced choice between two letters, only one of which appeared in the stimulus. In the Biederman (1972) and Biederman et al. (1974, Experiment II) studies, the subject was required to select the correct object-position pairing. Also, in the Biederman studies, superior recognition of the objects/relationships in the coherent condition over the recognition of these elements in the jumbled condition could provide a basis for superior response selection after imperfect perceptual processing.

\footnotetext{
(Received for publication February 3, 1975; revision accepted February 5, 1976.)
} 